\title{
Clara cells drive eosinophil accumulation in allergic asthma
}

\author{
S.S. Sonar*, M. Ehmke ${ }^{\#}$, L.M. Marsh", J. Dietze ${ }^{\#}$, J.C. Dudda ", M.L. Conrad ${ }^{\#}$, \\ H. Renz ${ }^{\#,+}$ and W.A. Nockher ${ }^{\#,+}$
}

ABSTRACT: Development of allergic asthma is a complex process involving immune, neuronal and tissue cells. In the lung, Clara cells represent a major part of the "immunomodulatory barrier" of the airway epithelium.

To understand the contribution of these cells to the inflammatory outcome of asthma, disease development was assessed using an adjuvant-free ovalbumin model. Mice were sensitised with subcutaneous injections of $10 \mu \mathrm{g}$ endotoxin-free ovalbumin in conjunction with naphthaleneinduced Clara cell depletion.

Clara epithelial cell depletion in the lung strongly reduced eosinophil influx, which correlated with decreased eotaxin levels and, moreover, diminished the T-helper cell type 2 inflammatory response, including interleukin (IL)-4, IL-5 and IL-13. In contrast, airway hyperresponsiveness was increased. Further investigation revealed Clara cells as the principal source of eotaxin in the lung.

These findings are the first to show that Clara airway epithelial cells substantially contribute to the infiltration of eotaxin-responsive CCR3+ immune cells and augment the allergic immune response in the lung. The present study identifies Clara cells as a potential therapeutic target in inflammatory lung diseases such as allergic asthma.

KEYWORDS: Airway epithelium, asthma, Clara cell, eosinophil, eotaxin, naphthalene

$\mathbf{A}$ llergic asthma is a chronic inflammatory disease characterised by airway hyperreactivity, eosinophilic inflammation, elevated serum immunoglobulin (Ig)E levels and mucus production. The disease is elicited by T-helper (Th) type 2 cells and their corresponding cytokines, such as interleukin (IL)-4, IL-5 and IL-13. Airway structure and integrity plays a critical role in pathogenesis as it serves to translate gene-environment interactions. Therefore, although initially considered as mainly a structural barrier, recent studies are focusing on the immunoregulatory function of the lung epithelium [1]. The airway epithelium comprises ciliated and secretory cells arranged in a continuous stratified structure. When affected by disease or injury, the epithelium can serve as an important source of autacoid mediators, chemokines and growth factors responsible for repair or sustaining ongoing inflammation. Of the epithelial cells lining the murine airways, Clara cells comprise the principal cell type, accounting for 70$90 \%$ of cells in distal airways [2]. In humans, Clara cells contribute substantially to cell renewal in the normal conducting airway epithelium [3,4]. The non-ciliated, secretory Clara cells express the IL-4 cytokine receptor [5] and can modulate immune responses by secretion of both pro- and antiinflammatory factors [6-8]. These features identify them as an important cell compartment linking innate and adaptive immune responses in the lungs. For example, Clara cells respond to activated Th2 cells via the IL-4 receptor [5], can differentiate into mucus-producing goblet cells [9] and secrete anti-inflammatory factors such as Clara cell secretory protein (CC10), which counter-regulates the Th2 response in asthma [10]. Clara cells are also able to metabolise and detoxify xenobiotics and toxic compounds such as naphthalene (NA) present in cigarette smoke, which enables their specific depletion as used in our study [11, 12].

Allergic lung inflammation involves the orchestrated recruitment of immune cells from the blood stream by tissue- and cell-specific signals, such as

This article has supplementary material available from www.erj.ersjournals.com

Note from the Editors: The term Clara cell is still used in the European Respiratory Journal (ERJ) and in other publications in the field. However, the use and possible replacement of this term has been the subject of some debate, as discussed in the ERJ article "The Clara cell: a "Third Reich eponym"?” by A. Winkelmann and T. Noak (Eur Respir J 2010; 36: 722-727).

\section{AFFILIATIONS}

*Institute of Integrative Biology, Molecular Biomedicine, ETH Zurich, Zurich,

"Ludwig Institute for Cancer Research, University of Lausanne, Lausanne, Switzerland.

\#Dept of Clinical Chemistry and Molecular Diagnostics, University Hospital Marburg, Marburg, Germany.

${ }^{+}$Both authors contributed equally.

CORRESPONDENCE

S.S. Sonar

Institute of Integrative Biology

Molecular Biomedicine

ETH Zurich

Wagistrasse 27

8952 Schlieren-Zurich

Switzerland

E-mail: sanchaita.sonar@env.ethz.ch

Received:

Dec 212011

Accepted after revision:

July 072011

First published online:

Aug 042011 
chemokines and adhesion molecules [13, 14]. Accumulation of eosinophils is a crucial event for the development of asthma and is found in the lungs, sputum and bronchoalveolar lavage of patients, and correlates well with asthma severity [15, 16]. Eosinophils secrete tissue-damaging cytotoxic granule cationic proteins, such as major basic protein (MBP), eosinophil cationic protein, eosinophil peroxidase and eosinophil-derived neurotoxin. In addition, eosinophils are major reservoirs of cytokines, chemokines, reactive oxygen species, arachidonic acid metabolites and other mediators that are involved in tissue inflammation [17]. Eosinophil recruitment is mediated by the chemokine eotaxin/CCL11 and its receptor CCR3, which is also expressed on other cells such as basophils, T-cells and microglial cells [1820]. The airway epithelium has been described as a prominent cellular source for chemokines and a few studies have demonstrated eotaxin expression by airway epithelial cells in vivo and in vitro, although the cell types expressing eotaxin have never been clearly identified [21, 22]. The aim of our study was to examine the role of airway epithelial Clara cells in the trafficking of inflammatory cells into allergic lungs. Using an established model of NA-induced Clara cell ablation in combination with ovalbumin (OVA)-driven asthma, the present study shows that Clara cells are crucial for the production of eotaxin and the accumulation of eosinophils in the airways.

\section{METHODS \\ Mice}

Female BALB/c mice aged 6-8 weeks were obtained from Harlan Winkelmann (Borchen, Germany). All experimental procedures were approved by the local animal ethics committee and met German and international guidelines. At least 8-10 animals were used per experimental group.

\section{Study design and animal treatment}

Mice were either sensitised with subcutaneous injections of $10 \mu \mathrm{g}$ endotoxin-free OVA (grade VI; Sigma-Aldrich, Hamburg, Germany) in $200 \mu \mathrm{L}$ PBS or sham injections of PBS on days 0,7 and 14 . The sensitisation phase was followed by 20 -min $1 \%$ OVA (grade V; Sigma) or sham PBS aerosol treatments on days 26, 27 and 28. Two additional groups of mice received OVA sensitisations as indicated above, along with one intraperitoneal injection $(200 \mu \mathrm{L})$ of NA $\left(200 \mathrm{mg} \cdot \mathrm{kg}^{-1}\right.$; Fluka, Hamburg, Germany) dissolved in corn oil (SigmaAldrich,) at day 3 (for analysis of AHR) or day 16 followed by OVA challenges as indicated above. Two additional groups of mice sensitised with OVA and further treated with NA $\left(200 \mathrm{mg} \cdot \mathrm{kg}^{-1}\right)$ received one single intranasal dose of recombinant murine eotaxin (4 $\mu \mathrm{g}$, CCL11; PeproTech, Rocky Hill, NJ, USA) at day 26 before challenge. Four additional control groups received sham injections of PBS on days 0,7 and 14, or corn oil or NA dissolved in corn oil at day 3 or day 16 (table 1).

\section{TUNEL assay}

To detect apoptotic cells in lung tissue sections, we utilised the TUNEL technique (DeadEND ${ }^{\mathrm{TM}}$ Fluorometric TUNEL System; Promega, Madison, WI, USA). Sections were deparaffinised and rehydrated, followed by pretreatment with $20 \mu \mathrm{g} \cdot \mathrm{mL}^{-1}$ proteinase K (Intergen Company, Purchase, NY, USA) for $10 \mathrm{~min}$ at room temperature. Slides were then fixed by immersing in $4 \%$ formaldehyde solution in PBS for $5 \mathrm{~min}$. This was followed by polymerisation of fluorescence-labelled uridine triphosphate on

\begin{tabular}{lcccccc} 
TABLE 1 & \multicolumn{6}{c}{ Experimental protocols utilised in this study } \\
Groups & PBS s.c. & OVA s.c. & PBS i.p. & CO i.p. & NA i.p. & ETX i.n. \\
\hline PBS/CO & + & - & - & + & - & - \\
PBS/NA & + & - & - & + & + & - \\
OVA/CO & - & + & - & + & - & - \\
OVA/NA & - & + & - & + & + & - \\
PBS/PBS & + & - & + & - & - & - \\
PBS/OVA & - & + & + & - & - & - \\
OVA/CO/ETX & - & + & - & + & - & + \\
OVA/NA/ETX & - & + & - & + & + & +
\end{tabular}

Groups included mice that were either sham-sensitised with PBS or ovalbumin (OVA)-sensitised, and treated with solvent corn oil (CO) only or with naphthalene (NA) dissolved in CO. Additional groups included OVA-sensitised CO- or NA-treated mice that received a single dose of intranasal eotaxin (ETX; $4 \mu \mathrm{g}$ ) on the first day of challenge. s.c.: subcutaneous; i.p.: intraperitoneal; i.n. intranasal. +: treated with; -: excluded.

nicked DNA ends as per manufacturer instructions. The sections were immediately analysed under a fluorescence microscope.

\section{Lung histology and immunohistochemistry}

Lungs were fixed with $10 \%$ formalin through the trachea, isolated and stored in $10 \%$ formalin. Lung tissues were embedded into paraffin and 3- $\mu \mathrm{m}$ sections were stained with haematoxylin and eosin (H\&E) or Periodic acid-Schiff (PAS) for light microscopy, as described elsewhere [23]. Immuno-histochemical staining was performed on paraffin-embedded lung tissue. Following rehydration in graded alcohol, tissue slices $(3 \mu \mathrm{m})$ were treated with citrate solution for antigen retrieval according to the manufacturer's instructions. Sections were incubated with antibodies recognising CC10, CCR3 (Abcam, Cambridge, UK) and CCL11 (R\&D Systems, Wiesbaden-Nordenstadt, Germany) overnight at $4{ }^{\circ} \mathrm{C}$. Peroxidase-conjugated anti-rabbit, anti-goat and anti-rabbit secondary antibodies (Vector Laboratories, Loerrach, Germany) were used for the detection of bound CC10, CCR3 and eotaxin, respectively. Diaminobenzidine (DAB) was used as chromomeric substrate (Vector Laboratories). For immunofluorescence analysis, anti-CC10 and anti-eotaxin antibodies were detected with Alexa Fluor 555 or Alexa Fluor 488 conjugated secondary antibodies (Invitrogen, Darmstadt, Germany), respectively. Sections were analysed using light microscopy (Olympus Europa $\mathrm{GmbH}$, Hamburg, Germany).

\section{Morphometric analysis of Clara cells and CCR3+ cells}

Mouse lungs were fixed by airway instillation using $6 \%$ phosphate-buffered paraformaldehyde at a pressure of $20 \mathrm{~cm}$ fluid column. Lung volume was determined by fluid displacement, and systemic uniform random samples of lung tissue were taken and processed according to standard methods. Immunohistochemical staining was performed on paraffin-embedded lung tissue, as described above. Briefly, sections were incubated with antibodies recognising CC10 (Upstate; Millipore, Billerica, MA, USA) overnight at $4^{\circ} \mathrm{C}$. Peroxidase-conjugated anti-rabbit secondary antibodies (Vector Laboratories) were used for the detection of bound CC10. DAB was used as chromomeric substrate (Vector Laboratories). The morphometric analysis for CC10 positive cells was performed using a modified version of a 
previously described method [24]. Proximal and distal airways were counted in a blinded manner. CC10 positive cells containing nuclear profiles were counted.

\section{Airway reactivity}

Airway hyperresponsiveness (AHR) was performed using the noninvasive head-out body plethysmography method, $24 \mathrm{~h}$ after the last aerosol challenge. The mid-expiratory airflow of bronchial responsiveness to $\beta$-methacholine was measured as described previously [25].

\section{Assessment of leukocyte distribution in bronchoalveolar lavage fluid}

$48 \mathrm{~h}$ after the last challenge, mice were sacrificed and bronchoalveolar lavage fluid (BALF) was generated as described
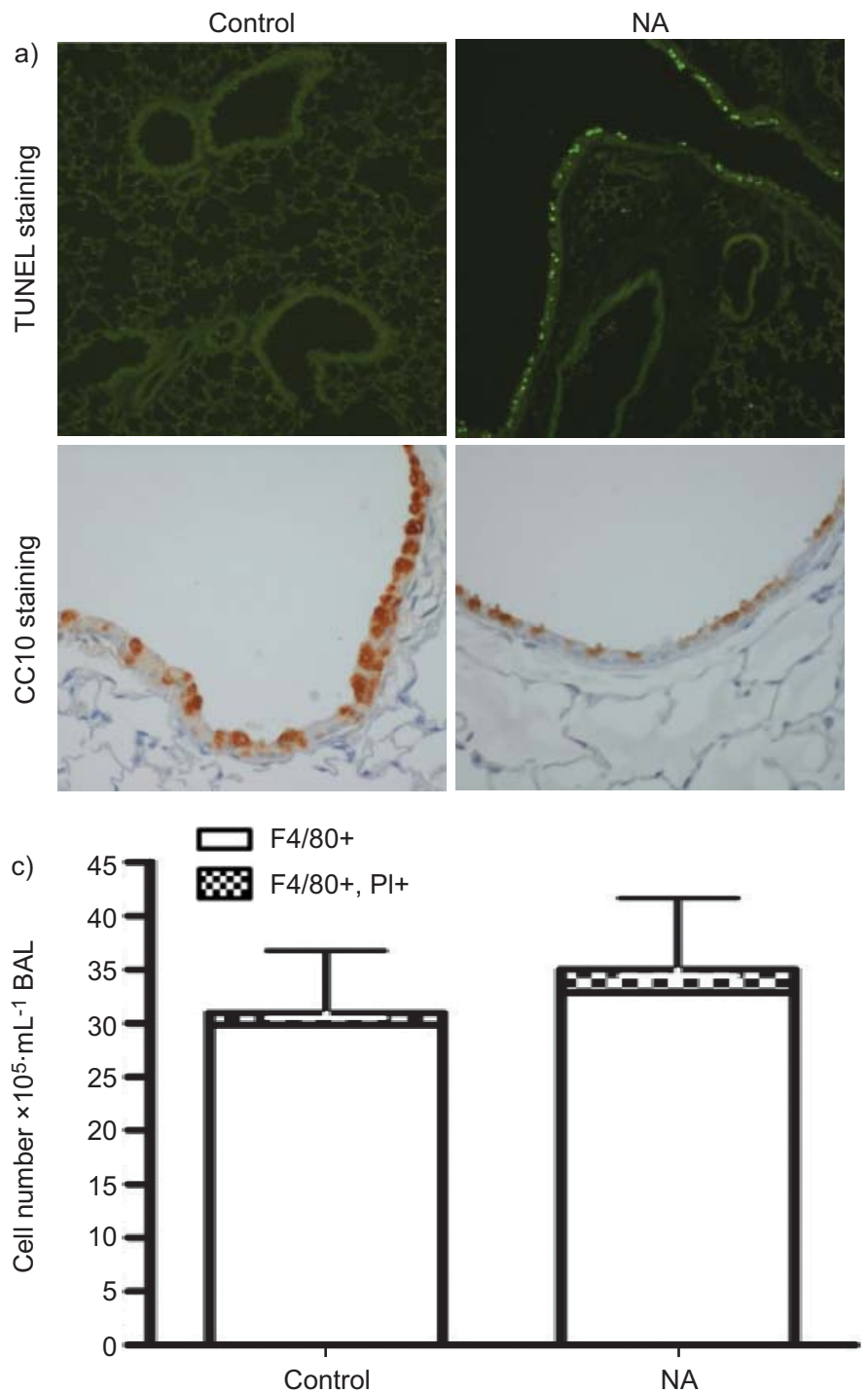

previously [23]. The total number of leukocytes was determined using a Casy TT Cell Counter (Schärfe System GmbH, Reutlingen, Germany). BALF cells were differentially stained with Diff-Quick (Dade Behring, Marburg, Germany) and numbers of lymphocytes, eosinophils and neutrophils were identified by standard morphologic criteria, with 100 cells counted per cytospin. Cell-free lavage fluids were stored at $-20^{\circ} \mathrm{C}$ for further cytokine analysis.

\section{Measurements of cytokine levels in BALF}

Cytokines including IL-1 $\alpha$, IL-2, IL-4, IL-5, IL-10, IL-13, interferon (IFN)- $\gamma$ and granulocyte-macrophage colony-stimulating factor (GM-CSF) were measured from the cell-free supernatants of the BALF according to the manufacturer's instructions (Th1/ Th2 10plex Flowcytomix kit and Mouse Chemokines 6plex FlowCytomix Multiplex; Bender MedSystems, Vienna, Austria).
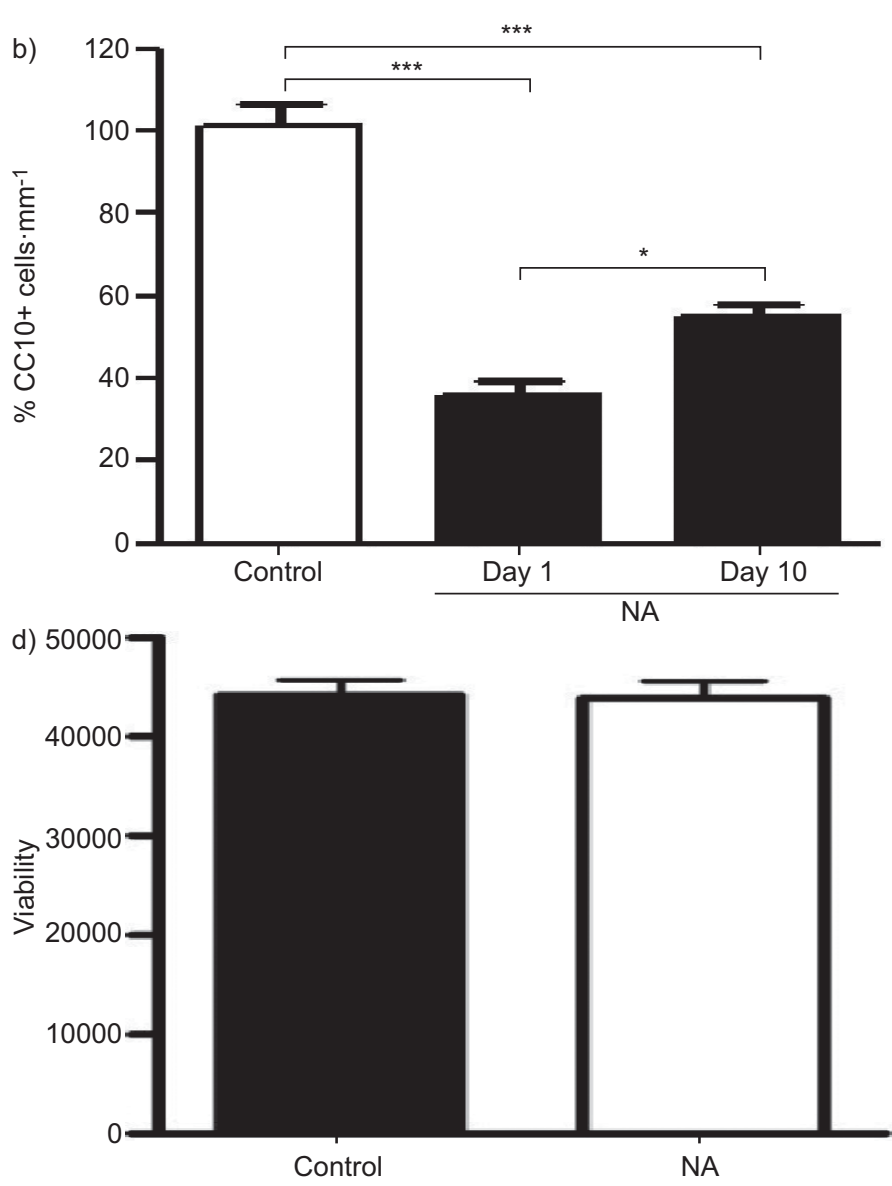

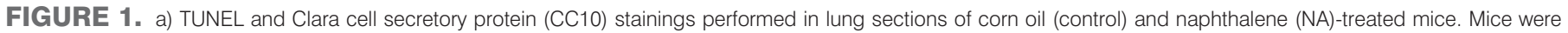

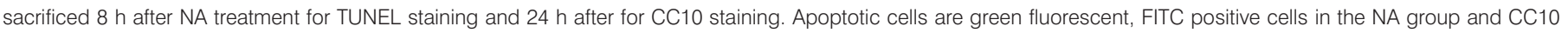

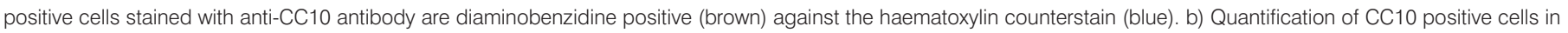

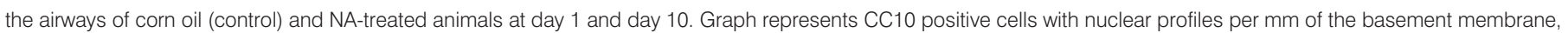

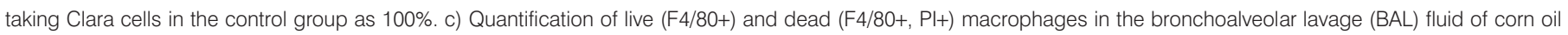

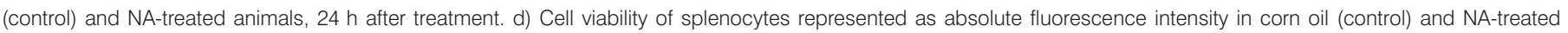
animals. Data are presented as mean \pm SEM of at least six animals. ${ }^{*}: p<0.05$; ${ }^{*} *: p<0.001$. Representative results for one out of three experiments. 
Eotaxin (CCL11) and thymic stromal lymphopoietin (TSLP) were detected using the Quantkine immunoassay (R\&D Systems) according to the manufacturer's instructions.

\section{Flow cytometry}

Fluorescence-activated cell sorting (FACS) was performed on cells isolated from the BALF to measure the total number of CD45+ leukocytes using antibodies to murine CD45 (clone 104; BD Biosciences, Heidelberg, Germany). The total number of leukocytes was calculated by extrapolating the percentage of CD45+ cells obtained by FACS analysis to absolute numbers, as counted by the electronic Casy TT Cell Counter (Schärfe System $\mathrm{GmbH}$ ). FACS was also performed to detect live and dead macrophages using the F4/80 antigen (clone 6F12; BD Biosciences) together with propidium iodide. CCR3+ cells were discriminated via CCR3 antibody surface staining (clone 83103; BD Biosciences) within CD45+ cells.

\section{Statistics}

Statistical significance for normally distributed samples was analysed using an unpaired t-test. Non-normal data or data with unequal variances were tested for significance using the
Mann-Whitney rank sum test. A p-value $<0.05$ was considered statistically significant. Results are presented as mean \pm SEM. Groups consisted of at least eight mice or six independent samples (in vitro) and experiments were repeated at least three times. Calculations were performed using GraphPad Prism Software, version 3.02 (www.graphpad.com).

\section{RESULTS \\ NA treatment selectively depletes Clara cells}

To assess the role of bronchial epithelial Clara cells in allergic asthma, BALB/c mice were subjected to OVA experimental asthma protocols with or without NA treatment (table 1). Treatment with NA resulted in apoptosis of bronchial epithelial cells as examined after $8 \mathrm{~h}$ using the TUNEL assay (fig. 1a). NA induced Clara cell-specific death without any other cellular alteration or nonspecific cell death in the lungs as reported previously [26-28]. Within $24 \mathrm{~h}$, extensive detachment of Clara cells from the basement membrane and almost complete denudation of the epithelium in some airways was observed (fig. 1a). Quantification of Clara cells using CC10-specific staining revealed $\sim 65 \%$ Clara cell depletion on day 1 ; further quantification at day 10 showed renewal of Clara cells to $\sim 50 \%$
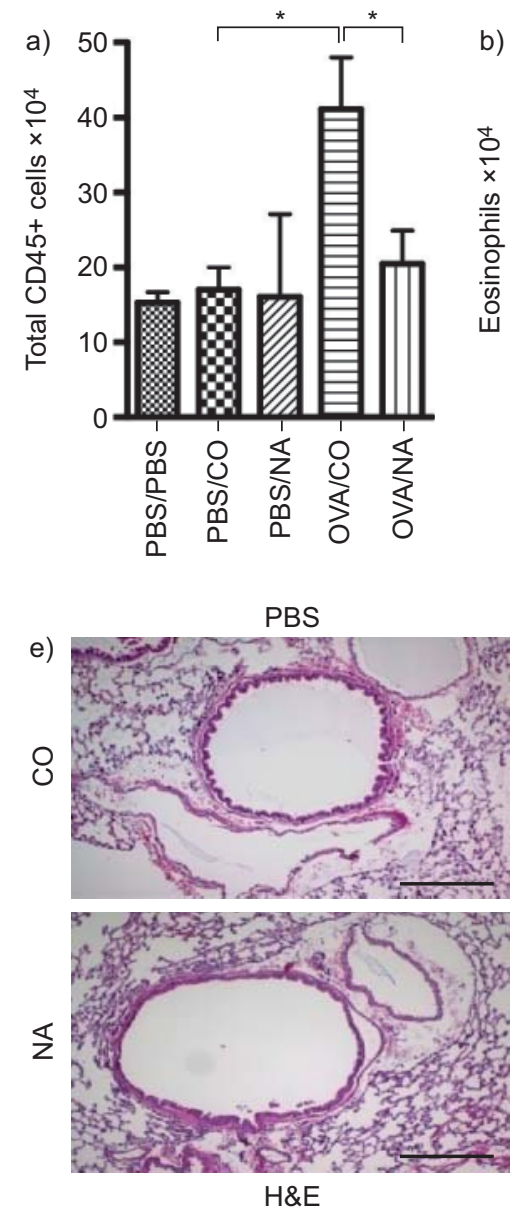
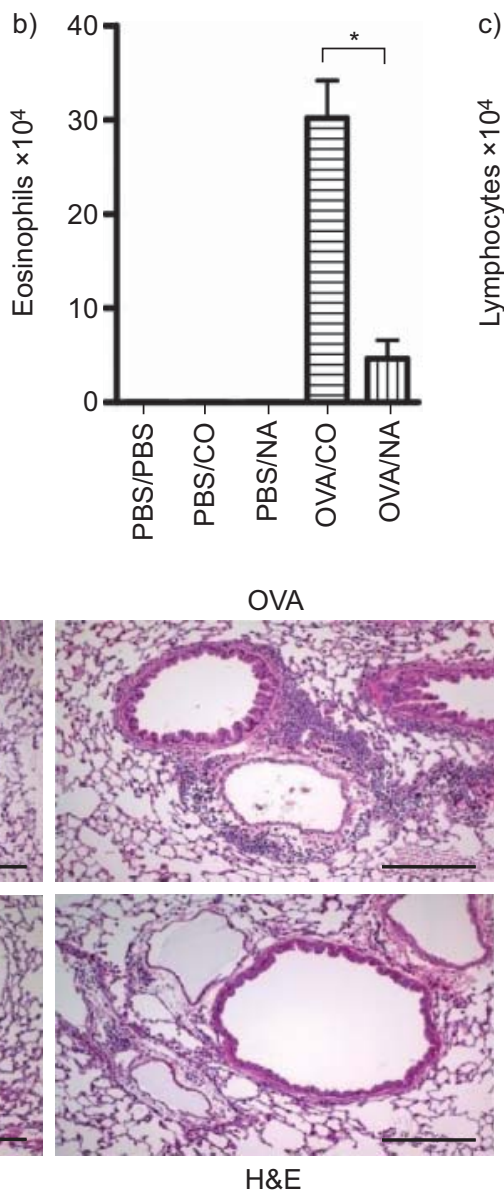
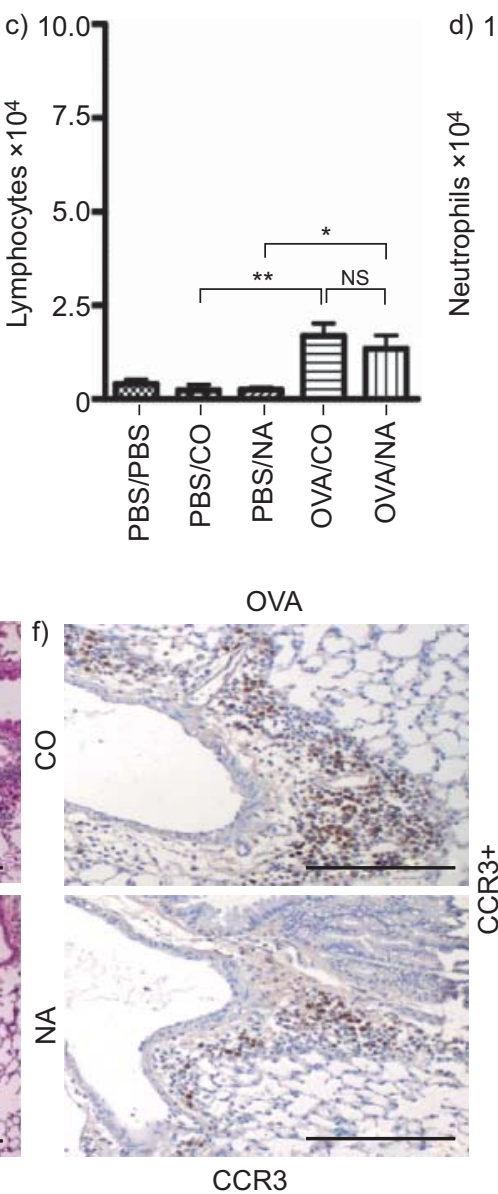
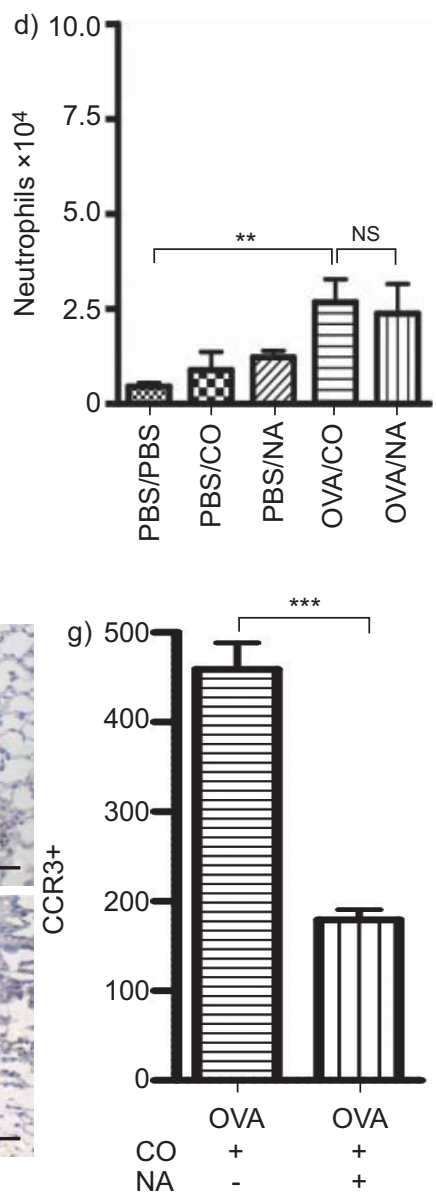

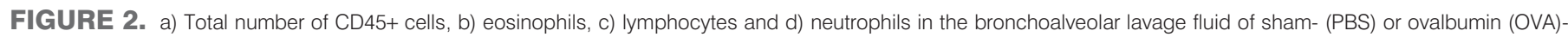

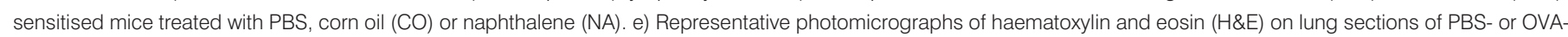

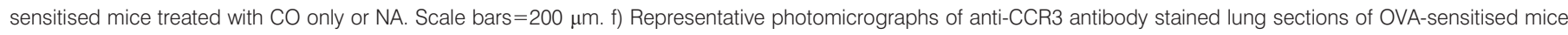

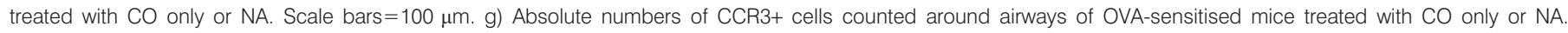
NS: nonsignificant. +: treated with; -: excluded. *: $p<0.05$; **: $p<0.01$; ***: $p<0.001$. 
(fig. 1b). Potential NA toxicity on other cell types was further investigated, testing alveolar macrophages and splenic mononuclear cells (MNC) isolated from mice $48 \mathrm{~h}$ after treatment. FACS analysis of BAL cells using the macrophage-specific cell surface glycoprotein marker F4/80 revealed no difference in the frequency of live (F4/80+, PI-) and apoptotic (F4/80+, PI+) cells between control and NA-treated groups (fig. 1c). Furthermore, no differences in the viability of MNC were observed between NA-treated and untreated animals (fig. 1d). These results suggest that NA is selectively toxic for Clara cells in the lung with no cytotoxic effects on other cell types.

Clara cell depletion suppresses allergic lung inflammation The NA model of Clara cell denudation was then employed to assess the role of Clara cells in allergic airway inflammation using an adjuvant-free model of asthma. Analysis of the total number of inflammatory cells in the BALF of OVA-sensitised and NA-treated mice revealed a strong reduction of CD45+ cells as compared with OVA-sensitised mice treated with solvent (corn oil) alone (fig. 2a). Examination of the differential cell types showed a specific reduction in the number of eosinophils (fig. 2b), which are the predominant inflammatory cell infiltrate in this model [23]. In contrast, the moderate increase seen in the numbers of lymphocytes and neutrophils in the BALF following allergen challenges was not affected by NA treatment (fig. 2c and d). Peribronchial eosinophil recruitment was then examined using H\&E and anti-CCR3 staining. Lung histology of OVAsensitised mice treated with corn oil revealed the typical peribronchial inflammation dominated by CCR3+ cells confirming the BALF results (fig. 2f). Again, the Clara cell-denuded, OVA-sensitised mice revealed a strong reduction specifically of CCR3+ cells in the inflammatory infiltrate surrounding the airways (fig. $2 \mathrm{f}$ and g). Non-sensitised control groups with or without subsequent treatment with NA did not exhibit any inflammation in the lung tissue (fig. 2e). Furthermore, after OVA sensitisation and challenge, goblet cell hyperplasia was induced in the bronchial epithelia; however, fewer PAS (for mucus producing cells) positive cells were present in the OVA/NAtreated groups due to denudation of Clara cells (data not shown).

Additionally, we were interested in the levels of the Clara cell-associated markers CC10 and surfactant protein (SP)-D. Induction of allergic asthma led to an increase in CC10 and SP-D mRNA levels when compared with PBS-treated control mice (fig. S1 B of the online supplementary material). However, NA treatment in OVA-sensitised mice showed a reduction in the levels of Clara cell-associated factors that was similar to control mice (fig. S1 B of the online supplementary material).

Together, these results demonstrate that Clara cells are an important immunoregulatory cell type in the lung and are responsible for the recruitment of eosinophils, but not other immune cells, to the airways in allergic asthma.

\section{Depletion of Clara cells increases AHR}

Next, we considered the effect of Clara cell depletion on lung function in allergic asthma and measured AHR from control and sensitised mice with or without Clara cell depletion on day 3 or day 16 after sensitisation. As expected, sensitisation to OVA strongly increased AHR, which was not significantly influenced by depletion of Clara cells at day 3 (fig. 3). Interestingly, depletion on day 16 further increased AHR

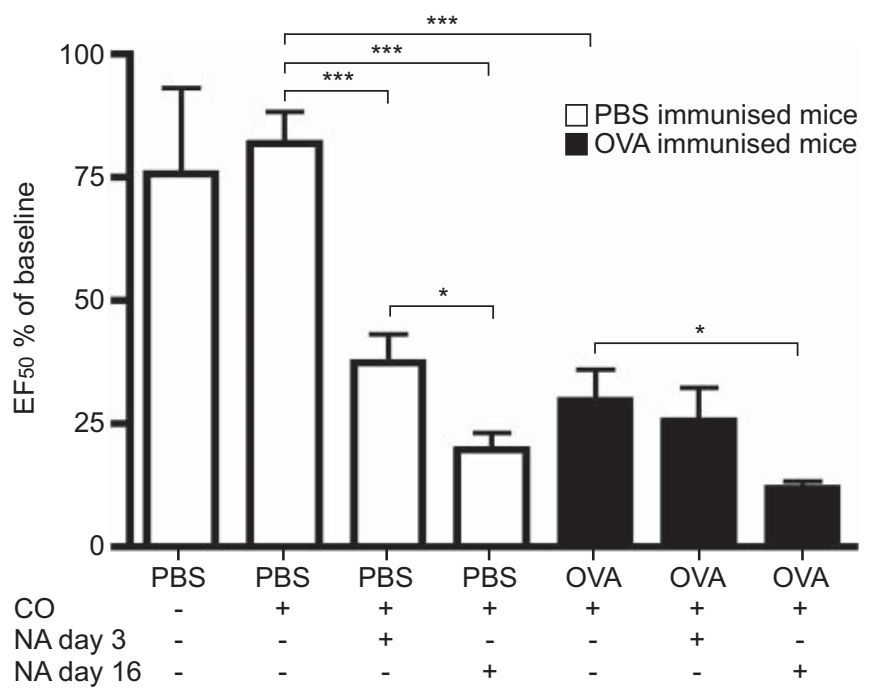

FIGURE 3. Airway reactivity to methacholine in PBS- and ovalbumin (OVA)immunised mice treated with either PBS, corn oil (CO) only or naphthalene (NA) at day 3 or day 16. Airway responsiveness to methacholine was analysed using headout body plethysmography $24 \mathrm{~h}$ after the last challenge with OVA. Data presented as mean \pm SEM of at least six animals. EF50: mid-expiratory airflow. +: treated with; -: excluded. ${ }^{*}: p<0.05 ; * \star *: p<0.001$.

compared with sensitised and CO-treated mice. These data suggest that disruption of airway epithelium makes it more responsive to reactive substances such as methacholine.

\section{Th2 cytokine and eotaxin production in allergic asthma depends on Clara cells}

The Th2-associated cytokines IL-4, IL-5 and IL-13 are central to the development of allergic asthma. To investigate the basis by which Clara cells affect the recruitment of inflammatory cells, cytokines were measured in BALF (fig. 4). OVA-sensitised mice exhibited high levels of IL-4, IL-5 and IL-13. In contrast, these cytokines in the Clara cell-depleted mice were reduced to background levels (fig. $4 \mathrm{a}-\mathrm{c}$ ). No significant differences in IFN- $\gamma$, IL-2, IL-10 or IL-1 $\alpha$ were observed between all groups (fig. $4 \mathrm{~d}-\mathrm{g}$ ). Analysis of GM-CSF, reported to be upregulated in asthma and involved in induction of eosinophilia [29], also revealed no significant differences between groups in our model (fig. 4h), whereas other inflammatory factors like RANTES and TSLP were not detectable (data not shown). Strongly elevated levels of eotaxin were observed in the OVA-sensitised group with intact epithelium (OVA/CO), whereas levels were reduced to background following Clara cell depletion (fig. 4i). These data demonstrate that the production of Th2 cytokines and eotaxin in allergic asthma is dependent on intact Clara cells.

\section{Clara cells are the major source of eotaxin in the lungs}

To investigate the source of eotaxin in the airways, immunohistology from serial lung sections was performed. Clara cells identified by CC10 also stained positive for eotaxin (fig. $5 \mathrm{a}$ and b). In contrast, very few, if any, CC10 negative cells were positive for eotaxin. The staining pattern was similar for PBStreated control animals as well as OVA-sensitised animals. In order to determine whether the reduction in eotaxin levels upon depletion of Clara cells was responsible for the decreased eosinophil numbers, we supplemented OVA-sensitised mice 

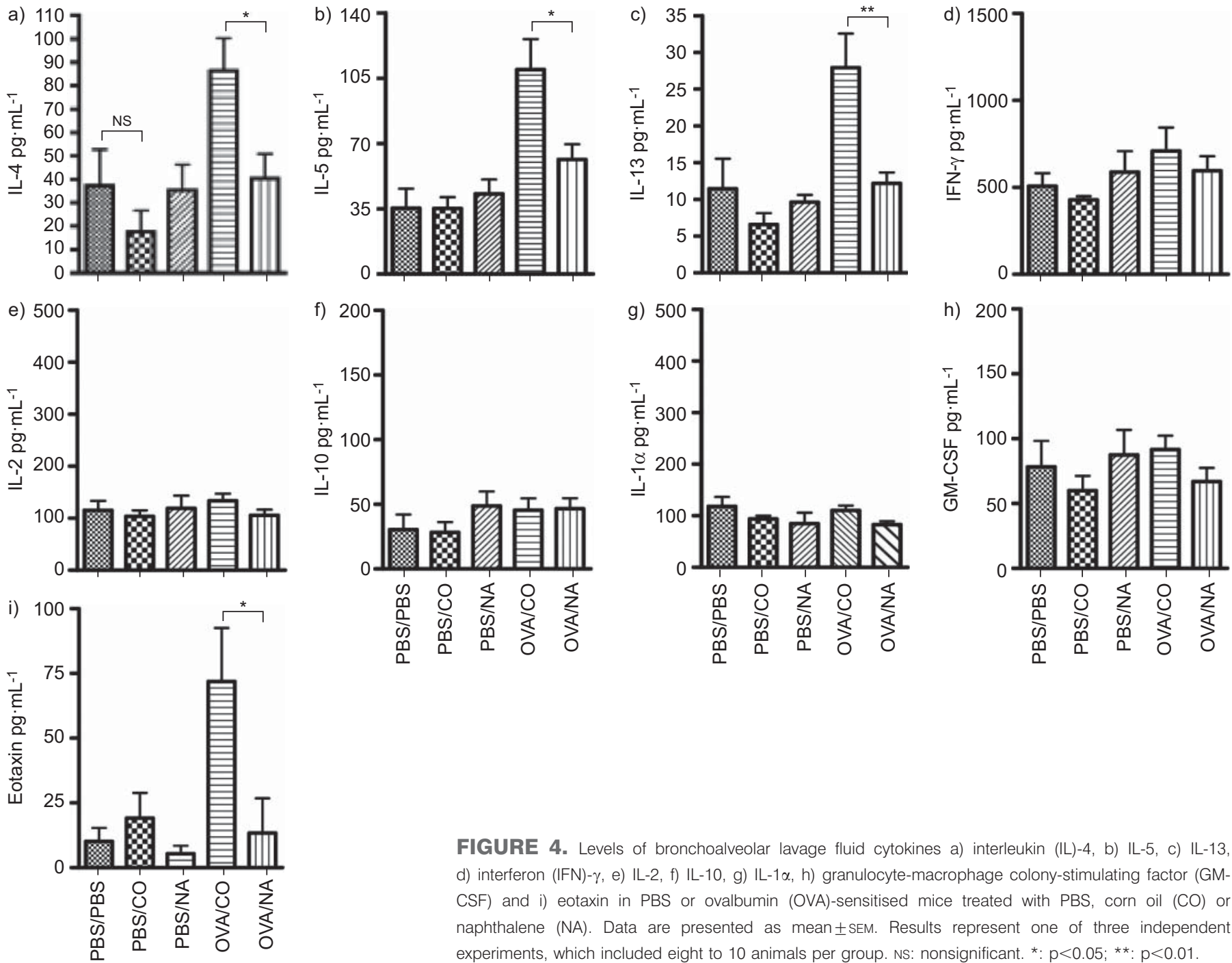

FIGURE 4. Levels of bronchoalveolar lavage fluid cytokines a) interleukin (IL)-4, b) IL-5, c) IL-13, d) interferon (IFN)- $\gamma$, e) IL-2, f) IL-10, g) IL-1 $\alpha$, h) granulocyte-macrophage colony-stimulating factor (GM$\mathrm{CSF}$ ) and i) eotaxin in PBS or ovalbumin (OVA)-sensitised mice treated with PBS, corn oil (CO) or naphthalene (NA). Data are presented as mean \pm SEM. Results represent one of three independent experiments, which included eight to 10 animals per group. NS: nonsignificant. ${ }^{*}: p<0.05$; ${ }^{* *}$ : $p<0.01$.

with one dose of eotaxin given on the first day of allergen challenge. Administration of eotaxin i.n. induced a significant increase in CCR3+ cells in the lungs of OVA-sensitised as well as Clara cell-depleted mice (fig. $5 c$ and d). These results suggest that Clara cell-derived eotaxin is crucial for eosinophil accumulation in allergic lung inflammation. Furthermore, analysis of IL-4, IL-5, IL-13 and IFN- $\gamma$ in Clara cell-depleted OVA-sensitised animals revealed an increase in the Th2 cytokines following eotaxin supplementation (fig. 6). IL-4 and IL-13 levels increased independently of eotaxin supplementation (fig. 6a and c), whereas IL-5 production increased only after eotaxin application in Clara cell-depleted mice (fig. 6b). IFN- $\gamma$ levels were low and did not show significant changes (fig. 6d). Additionally, administration of eotaxin in OVAsensitised mice did not affect CC10 or SP-D expression (fig. S1B of the online supplementary material).

These results suggest that Clara cells are the main producers of eotaxin in the lung under steady-state and inflammatory conditions, and are therefore responsible for the recruitment of eosinophils in allergic inflammation.

\section{DISCUSSION}

We have demonstrated that Clara cell-derived eotaxin is crucial for the accumulation of eosinophils during allergic lung inflammation. Clara cells have previously been reported to secrete various factors that are involved in the protection and defence mechanisms of the lung. For example, CC10 and SP-D secreted by Clara cells play a protective role in asthma by inhibiting Th2 responses [10, 30]. Pro-inflammatory mediators, such as acidic mammalian chitinase, are upregulated in Clara cells during Th2 inflammation and their inhibition ameliorates inflammation [31]. Chemokines, such as keratinocyte chemoattractant and macrophage inflammatory protein- $1 \alpha$, are secreted by Clara cells and their release following lipopolysaccharide application upon Clara cell ablation has been found to be significantly reduced [7]. Additionally, the expression of pattern recognition receptors, such as Toll-like receptor 4 , on the lung epithelium has a critical role for the activation of dendritic cells (DC) in allergic lung inflammation, stressing the importance of the lung tissue microenvironment for efficient $\mathrm{T}$ cell activation [32]. Moreover, several reports have described the modulation of gene expression in Clara cells by the Th2 

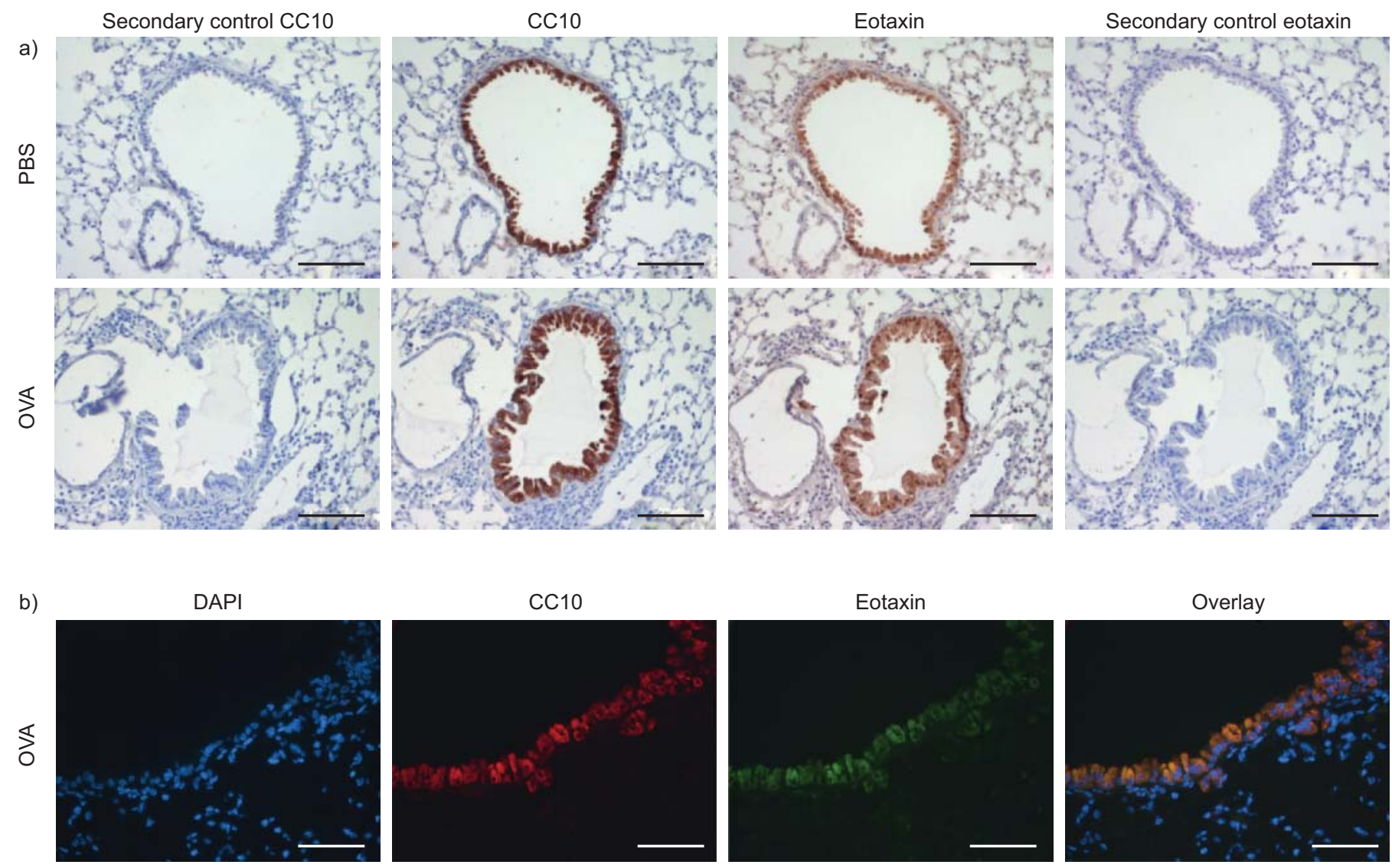

c)

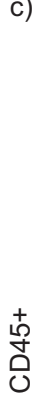

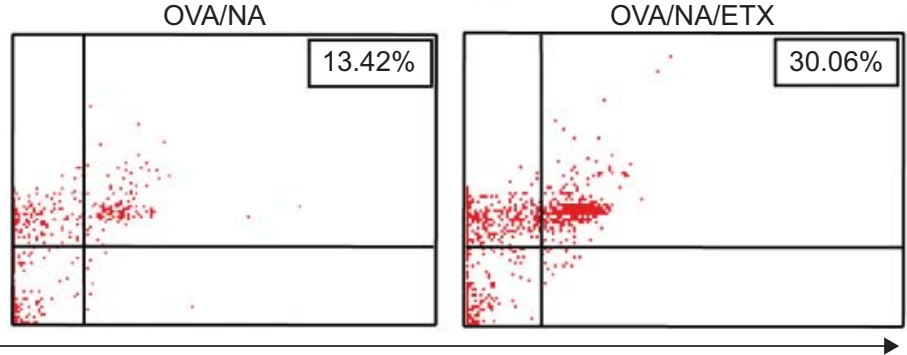

OVA/CO/ETX

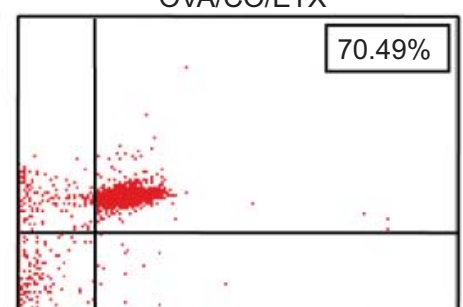

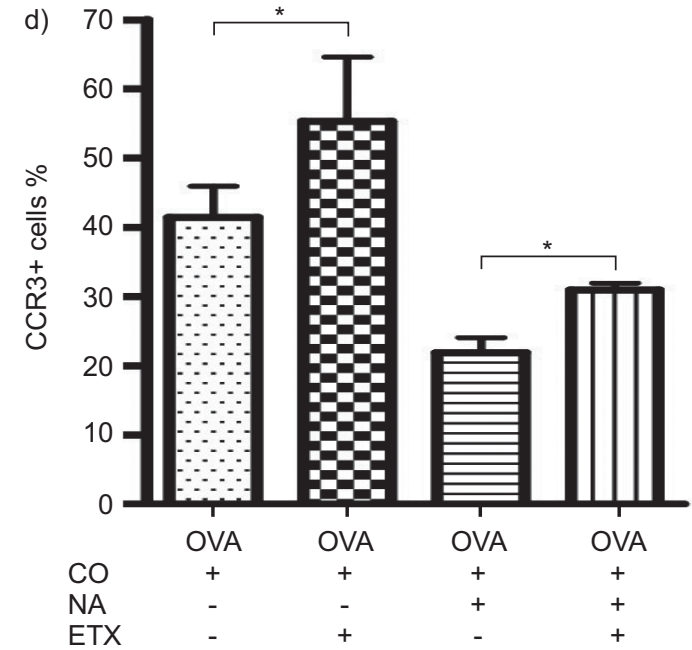

FIGURE 5. a) Serial sections of control (PBS) and ovalbumin (OVA)-sensitised animals stained for Clara cell secretory protein (CC10) and eotaxin. Groups represent secondary antibody control for CC10, specific staining for CC10, eotaxin and secondary antibody control for eotaxin. Both CC10 positive Clara cells and eotaxin-specific staining are diaminobenzidine positive (brown) against the haematoxylin (blue) counter stain. Scale bars $=100 \mu \mathrm{m}$. b) Immunofluorescence staining performed on serial sections from OVA-sensitised animals for DAPI nuclear stain, CC10, eotaxin and their overlay images. c) Fluorescence-activated cell sorting analysis of CD45+/CCR3+ cells derived from the bronchoalveolar lavage fluid of OVA-sensitised animals either treated with corn oil (CO) alone or naphthalene (NA) with or without intranasal eotaxin (ETX). d) Quantification of CCR3+ cell percentage as analysed in c. Data are presented as mean \pm SEM for eight to 10 animals in each group of mice. Representative results for one out of three experiments. +: treated with; -: excluded. *: $p<0.05$. 

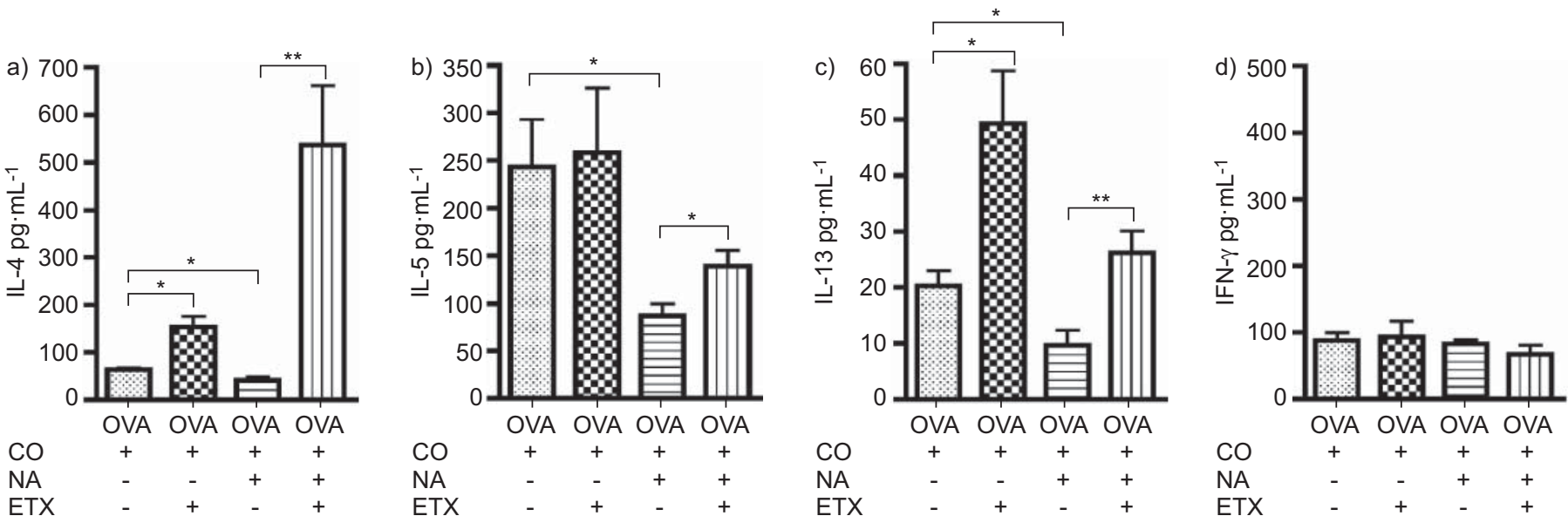

FIGURE 6. Levels of bronchoalveolar lavage fluid cytokines a) interleukin (IL)-4, b) IL-5, c) IL-13 and d) interferon (IFN)- $\gamma$ in ovalbumin (OVA)-sensitised mice either treated with corn oil (CO) alone or naphthalene (NA) with or without intranasal eotaxin (ETX) application. Data are presented as mean \pm SEM for eight to 10 animals in each group of mice. Representative results for one out of three experiments. +: treated with; -: excluded. *: $p<0.05 ;{ }^{*}$ : $p<0.01$.

cytokines IL-4 and IL-13 [33, 34]. Ablation of nuclear factor- $\kappa B$ signalling in Clara cells reduces peribronchial fibrosis, CD4+ cell infiltration, airway mucus, CCL17 and eotaxin release and eosinophils in allergen-challenged mice [34, 35]. Together, these and other studies demonstrate that epithelial cells have the ability to respond and orchestrate lung immune responses. However, knowledge about the specific contribution of the epithelia cell subset to these processes is still incomplete.

In humans, the number of Clara cells in the terminal and respiratory bronchioles has been reported to be $\sim 11$ and $22 \%$, respectively [3]. Clara cells are important for regeneration and differentiation into other epithelial cell types [36], e.g. goblet cells, which are responsible for mucus production and comprise a portion of CC10 immunoreactive bronchial cells [3]. Investigation in smokers revealed a significant decrease in the proportion of bronchiolar Clara cells, in contrast to nonsmoking patients, indicating that Clara cell numbers can be affected by inhaled agents [37]. However, Clara cells have not been specifically investigated in lung diseases like asthma, where the airway epithelium plays a key role in sensitisation to innocuous agents. In fact, progressive epithelial damage has been described in chronic cases of human asthma and although generally regarded as a disease of the larger airways, smaller distal airways, where Clara cells are prominent, have been increasingly implicated in the pathogenesis of allergic asthma [38, 39].

To investigate the role of Clara cells, we used NA to selectively deplete these cells from the airways of mice. NA is a polycyclic aromatic hydrocarbon commonly found in cigarette smoke and diesel exhaust that is capable of inducing a highly dosedependent, cell type- and site-selective toxicity [40]. Toxicity requires metabolic activation, which is catalysed by cytochrome P450 mono-oxygenases. NA has been reported to selectively injure the nonciliated Clara cells, since they are the primary expression site for cytochrome P450 monooxygenase 2F2, an isoform with high catalytic activity for NA [41].

The experiments presented here support previous findings that NA-treatment efficiently induces apoptosis in the majority of Clara cells with no obvious cytotoxic side-effects on other cell types [26-28]. Importantly, mice with impaired Clara cells at the time of aerosol challenge showed strongly diminished eosinophil accumulation around the airways and in the BALF. Eotaxin is an important homing and survival factor for eosinophils, and strongly reduced eotaxin levels were detected in the BALF of Clara cell-depleted mice, suggesting that Clara cells participate in eotaxin production. In contrast, GM-CSF, another survival factor for eosinophils, was unaffected by Clara cell depletion. In fact, we could show for the first time that Clara cells are the major source of eotaxin, accounting for the moderate levels under steady-state conditions as well as the strongly increased release during inflammation. Although eosinophils were strongly reduced in Clara cell-depleted mice, numbers of Tcells and neutrophils remained unchanged, indicating that these cells obtained sufficient priming. One may speculate, however, that the lung DC may not have been appropriately recruited or triggered in the affected epithelium, as suggested by recently published results [32]. That T-cells and neutrophil accumulation was unaffected by Clara cell depletion indicates that these cells may utilise homing pathways independent of Clara cells. This is especially true for cell migration from tissue into BALF across the Clara cell epithelium. The similar inflammatory profile both in the tissue and the BALF, again suggests that migration of eosinophils was undisturbed in both compartments upon Clara cell depletion, but that eosinophil recruitment from the blood or their survival was affected in the absence of Clara cells. Although early Th2 cell recruitment was reported to be in part dependent on eotaxin [42], we did not observe differences in T-cell numbers upon Clara cell depletion.

Moreover, Clara cell depletion increased AHR, especially when Clara cells were depleted at a later time-point; 10 days before OVA lung challenge (day 16) rather than 23 days (day 3) before OVA lung challenge, suggesting an improvement in lung function with/upon regeneration of Clara cells. The fact that Clara cell depletion without sensitisation to OVA already induced AHR underlines the importance of the epithelium barrier for lung function and might be relevant for asthma 
patients exposed to substances that are likely to affect Clara cells, such as cigarette smoke and other abrasive pollutants in the environment.

We also detected strongly diminished amounts of IL-4, IL-13 and the eosinophil-promoting IL-5 in the BALF of Clara celldepleted mice. Supplementation with eotaxin in OVA-sensitised and CC-depleted lungs restored these cytokines along with eosinophil numbers. Interestingly, eotaxin was also able to boost higher Th2 cytokine production in intact airways, which was in line with an increase in eosinophil numbers in OVA sensitised/ challenged animals. As eosinophils are a major source for Th2 cytokines [43], the decrease in the Th2 cytokines upon Clara cell depletion and restoration after eotaxin application can be explained by the change in eosinophil numbers upon Clara cell depletion and eotaxin supplementation, respectively. Although Clara cells have been shown to express the IL- 4 and the IL-13 receptor [5], production of the corresponding Th2 cytokines from the airway epithelium is not known.

CC10 and SP-D are expressed by Clara cells and have been reported to play a role in allergic asthma; accordingly, we found them to be increased following OVA-sensitisation on RNA level. Depletion of Clara cells decreased the levels of both CC10 and SP-D, while eotaxin supplementation had no effect (fig. S1B and $\mathrm{C}$ of the online supplementary material). This was expected since the responsive eotaxin receptor CCR3 is not known to be expressed on lung epithelial cells. As we could restore eosinophil numbers and Th2 cytokines by supplementation with eotaxin in Clara cell-depleted mice, the lower CC10 and SP-D levels were unlikely to strongly affect the results in our experiments.

In summary, we have demonstrated that Clara cells are a major source of eotaxin in the airway epithelium and are crucial for orchestrating the allergic asthmatic response. In light of the important role played by the structural compartment to disease pathogenesis, recent clinical research increasingly focuses on therapies that interfere with tissue-specific homing pathways. The findings presented here provide further knowledge for future strategies involved in targeting the lung epithelium for therapy in allergic asthma.

\section{SUPPORT STATEMENT}

This work was supported in part by grants from the Deutsche Forschungsgemeinschaft (Transregio 22, Project A9) and the Stiftung für Pathobiochemie und Molekulare Diagnostik, DGKL.

\section{STATEMENT OF INTEREST}

None declared.

\section{ACKNOWLEDGEMENTS}

We thank A. Kilıç for valuable feedback and discussion about this paper, and A. Spies (both Dept of Clinical Chemistry and Molecular Diagnostics, University Hospital Marburg, Marburg, Germany) for technical assistance.

\section{REFERENCES}

1 Holgate ST. The epithelium takes centre stage in asthma and atopic dermatitis. Trends Immunol 2007; 28: 248-251.

2 Plopper CG, Suverkropp C, Morin D, et al. Relationship of cytochrome P-450 activity to Clara cell cytotoxicity. I. Histopathologic comparison of the respiratory tract of mice, rats and hamsters after parenteral administration of naphthalene. J Pharmacol Exp Ther 1992; 261: 353-363.
3 Boers JE, Ambergen AW, Thunnissen FB. Number and proliferation of clara cells in normal human airway epithelium. Am J Respir Crit Care Med 1999; 159: 1585-1591.

4 Shijubo N, Itoh Y, Yamaguchi T, et al. Clara cell protein-positive epithelial cells are reduced in small airways of asthmatics. Am J Respir Crit Care Med 1999; 160: 930-933.

5 Kuperman DA, Huang X, Nguyenvu L, et al. IL-4 receptor signaling in Clara cells is required for allergen-induced mucus production. J Immunol 2005; 175: 3746-3752.

6 Elizur A, Adair-Kirk TL, Kelley DG, et al. Clara cells impact the pulmonary innate immune response to LPS. Am J Physiol Lung Cell Mol Physiol 2007; 293: L383-L392.

7 Elizur A, Adair-Kirk TL, Kelley DG, et al. Tumor necrosis factoralpha from macrophages enhances LPS-induced clara cell expression of keratinocyte-derived chemokine. Am J Respir Cell Mol Biol 2008; 38: 8-15.

8 Park MS, Zhao B, Ramsay PL, et al. Expression of inflammatory cytokines in a mouse transformed Clara cell line by tumor necrosis factor-alpha. Ann N Y Acad Sci 2000; 923: 336-337.

9 Evans CM, Williams OW, Tuvim MJ, et al. Mucin is produced by clara cells in the proximal airways of antigen-challenged mice. Am J Respir Cell Mol Biol 2004; 31: 382-394.

10 Wang SZ, Rosenberger CL, Espindola TM, et al. CCSP modulates airway dysfunction and host responses in an Ova-challenged mouse model. Am J Physiol Lung Cell Mol Physiol 2001; 281: L1303-L1311.

11 Buckpitt A, Boland B, Isbell M, et al. Naphthalene-induced respiratory tract toxicity: metabolic mechanisms of toxicity. Drug Metab Rev 2002; 34: 791-820.

12 Chichester $\mathrm{CH}$, Buckpitt AR, Chang A, et al. Metabolism and cytotoxicity of naphthalene and its metabolites in isolated murine Clara cells. Mol Pharmacol 1994; 45: 664-672.

13 Busse WW, Banks-Schlegel S, Wenzel SE. Pathophysiology of severe asthma. J Allergy Clin Immunol 2000; 106: 1033-1042.

14 Hyun YM, Lefort CT, Kim M. Leukocyte integrins and their ligand interactions. Immunol Res 2009; 45: 195-208.

15 Bradley BL, Azzawi M, Jacobson M, et al. Eosinophils, Tlymphocytes, mast cells, neutrophils, and macrophages in bronchial biopsy specimens from atopic subjects with asthma: comparison with biopsy specimens from atopic subjects without asthma and normal control subjects and relationship to bronchial hyperresponsiveness. J Allergy Clin Immunol 1991; 88: 661-674.

16 Garcia-Zepeda EA, Rothenberg ME, Ownbey RT, et al. Human eotaxin is a specific chemoattractant for eosinophil cells and provides a new mechanism to explain tissue eosinophilia. Nat Med 1996; 2: 449-456.

17 Rothenberg ME. Eotaxin. An essential mediator of eosinophil trafficking into mucosal tissues. Am J Respir Cell Mol Biol 1999; 21: 291-295.

18 He J, Chen Y, Farzan M, et al. CCR3 and CCR5 are co-receptors for HIV-1 infection of microglia. Nature 1997; 385: 645-649.

19 Sallusto F, Mackay CR, Lanzavecchia A. Selective expression of the eotaxin receptor CCR3 by human T helper 2 cells. Science 1997; 277: 2005-2007.

20 Uguccioni M, Mackay CR, Ochensberger B, et al. High expression of the chemokine receptor CCR3 in human blood basophils. Role in activation by eotaxin, MCP-4, and other chemokines. J Clin Invest 1997; 100: 1137-1143.

21 Cook EB, Stahl JL, Lilly CM, et al. Epithelial cells are a major cellular source of the chemokine eotaxin in the guinea pig lung. Allergy Asthma Proc 1998; 19: 15-22.

22 Komiya A, Nagase H, Yamada H, et al. Concerted expression of eotaxin-1, eotaxin-2, and eotaxin-3 in human bronchial epithelial cells. Cell Immunol 2003; 225: 91-100.

23 Conrad ML, Yildirim AO, Sonar SS, et al. Comparison of adjuvant and adjuvant-free murine experimental asthma models. Clin Exp Allergy 2009; 39: 1246-1254. 
24 Stevens TP, McBride JT, Peake JL, et al. Cell proliferation contributes to PNEC hyperplasia after acute airway injury. Am J Physiol 1997; 272: L486-L493.

25 Glaab T, Daser A, Braun A, et al. Tidal midexpiratory flow as a measure of airway hyperresponsiveness in allergic mice. Am J Physiol Lung Cell Mol Physiol 2001; 280: L565-L573.

26 Plopper CG, Macklin J, Nishio SJ, et al. Relationship of cytochrome P-450 activity to Clara cell cytotoxicity. III. Morphometric comparison of changes in the epithelial populations of terminal bronchioles and lobar bronchi in mice, hamsters, and rats after parenteral administration of naphthalene. Lab Invest 1992; 67: 553-565.

27 Sonar SS, Schwinge D, Kilic A, et al. Nerve growth factor enhances Clara cell proliferation after lung injury. Eur Respir J 2010; 36: 105-115.

28 Yildirim AÖ, Veith M, Rausch T, et al. Keratinocyte growth factor protects against Clara cell injury induced by naphthalene. Eur Respir J 2008; 32: 694-704.

29 Stampfli MR, Wiley RE, Neigh GS, et al. GM-CSF transgene expression in the airway allows aerosolized ovalbumin to induce allergic sensitization in mice. J Clin Invest 1998; 102: 1704-1714.

30 Brandt EB, Mingler MK, Stevenson MD, et al. Surfactant protein D alters allergic lung responses in mice and human subjects. J Allergy Clin Immunol 2008; 121: 1140-1147.

31 Yang CJ, Liu YK, Liu CL, et al. Inhibition of acidic mammalian chitinase by RNA interference suppresses ovalbumin-sensitized allergic asthma. Hum Gene Ther 2009; 20: 1597-1606.

32 Hammad H, Chieppa M, Perros F, et al. House dust mite allergen induces asthma via Toll-like receptor 4 triggering of airway structural cells. Nat Med 2009; 15: 410-416.

33 Kim S, Shim JJ, Burgel PR, et al. IL-13-induced Clara cell secretory protein expression in airway epithelium: role of EGFR signaling pathway. Am J Physiol Lung Cell Mol Physiol 2002; 283: L67-L75.
34 Yamada A, Sheikh F, Niimi T, et al. Induction of uteroglobinrelated protein 2 (Ugrp2) gene expression by the Th2 cytokines IL-4 and IL-13. J Immunol 2005; 175: 5708-5715.

35 Poynter ME, Irvin CG, Janssen-Heininger YM. A prominent role for airway epithelial NF-kappa B activation in lipopolysaccharideinduced airway inflammation. J Immunol 2003; 170: 6257-6265.

36 Reynolds SD, Malkinson AM. Clara cell: progenitor for the bronchiolar epithelium. Int J Biochem Cell Biol 2010; 42: 1-4.

37 Shijubo N, Itoh Y, Yamaguchi T, et al. Serum and BAL Clara cell 10 $\mathrm{kDa}$ protein (CC10) levels and CC10-positive bronchiolar cells are decreased in smokers. Eur Respir J 1997; 10: 1108-1114.

38 Corren J. Small airways disease in asthma. Curr Allergy Asthma Rep 2008; 8: 533-539.

39 Contoli M, Bousquet J, Fabbri LM, et al. The small airways and distal lung compartment in asthma and COPD: a time for reappraisal. Allergy 2010; 65: 141-151.

40 Van Winkle LS, Buckpitt AR, Nishio SJ, et al. Cellular response in naphthalene-induced Clara cell injury and bronchiolar epithelial repair in mice. Am J Physiol 1995; 269: L800-L818.

41 Fanucchi MV, Murphy ME, Buckpitt AR, et al. Pulmonary cytochrome P450 monooxygenase and Clara cell differentiation in mice. Am J Respir Cell Mol Biol 1997; 17: 302-314.

42 Lloyd CM, Delaney T, Nguyen T, et al. CC chemokine receptor (CCR)3/eotaxin is followed by CCR4/monocyte-derived chemokine in mediating pulmonary $\mathrm{T}$ helper lymphocyte type 2 recruitment after serial antigen challenge in vivo. J Exp Med 2000; 191: 265-274.

43 Spencer LA, Szela CT, Perez SA, et al. Human eosinophils constitutively express multiple Th1, Th2, and immunoregulatory cytokines that are secreted rapidly and differentially. J Leukoc Biol 2009; 85: 117-123. 